\title{
Vaccinomics for the Major Blood Feeding Helminths of Humans
}

\author{
Alex Loukas, Soraya Gaze, Jason P. Mulvenna, Robin B. Gasser, ${ }^{2}$ Paul J. Brindley, ${ }^{3}$ Denise L. Doolan, ${ }^{4}$ \\ Jeffrey M. Bethony, ${ }^{3}$ Malcolm K. Jones, ${ }^{5}$ Geoffrey N. Gobert, ${ }^{4}$ Patrick Driguez, ${ }^{4}$ \\ Donald P. McManus, ${ }^{4}$ and Peter J. Hotez ${ }^{3}$
}

\begin{abstract}
Approximately one billion people are infected with hookworms and/or blood flukes (schistosomes) in developing countries. These two parasites are responsible for more disability adjusted life years lost than most other neglected tropical diseases (NTDs), and together, are second only to malaria. Although anthelmintic drugs are effective and widely available, they do not protect against reinfection, resistant parasites are likely to emerge, and mass drug administration programs are unsustainable. Therefore, there is a pressing need for the development of vaccines against these parasites. In recent years, there have been major advances in our understanding of hookworms and schistosomes at the molecular level through the use of "omics" technologies. The secretomes of these parasites have been characterized using transcriptomics, genomics, proteomics, and newly developed gene manipulation and silencing techniques, and the proteins of interest are now the target of novel antigen discovery approaches, notably immunomics. This research has resulted in the discovery, development, and early stage clinical trials of subunit vaccines against hookworms and schistosomes.
\end{abstract}

\section{Introduction}

$\mathbf{T}$ HE GENOMICS REVOLUTION has enormous implications for public health (Burke et al., 2010). In this review we focus on recent advances in the "omics" for neglected tropical diseases (NTDs), and discuss how these advances will facilitate the development of new and sustainable control methods for NTDs. Hookworms and blood flukes (schistosomes) are some the most important parasites of humans in terms of their global health impact on children, pregnant women, and people engaged in subsistence farming (Hotez et al., 2008a, 2008b). Together, their disease burdens exceed those of all other NTDs (Hotez et al., 2010). Because hookworms and schistosomes typically impact human health without resulting in mortality, they have not gotten the full attention of the global health policymakers and have not been prioritized either for large-scale control efforts or for research funding. However, when the chronic morbidities due to hookworm disease and schistosomiasis are fully considered based on disability-adjusted life years (DALYs) lost, these diseases together rank among the most important in developing countries, resulting in an annual loss of between 4.5 and 92 million
DALYs (Hotez et al., 2010; King and Dangerfield-Cha, 2008). Both infections/diseases can be treated with anthelmintic drugs, but this approach does not protect against the high frequency of reinfection with the parasites (Hotez et al., 2010). Moreover, single-dose mebendazole has been shown to exhibit poor efficacy for hookworm and after repeated administration in the same endemic population (Keiser and Utzinger, 2008), efficacy has been reported to diminish over time (Albonico et al., 2003), raising concerns about possible drug resistance. Indeed, drug failures have arisen with benzimidazoles in livestock (Geerts and Gryseels, 2000). Importantly, the mainstay of control for schistosomiasis is the quinoline compound, praziquantel (PZQ). There are concerns that mass administration of PZQ is unsustainable because of lower than expected efficacies of single-dose PZQ, and high rates of reinfection after treatment, pointing toward the potential emergence of anthelminthic drug resistance (Clements et al., 2009). Indeed, PZQ resistance has already been documented in Schistosoma mansoni in the laboratory (Fallon and Doenhoff, 1994). Mass drug administration programs are therefore inadequate in isolation, cannot hope to advance global elimination efforts, and new tools and

\footnotetext{
${ }^{1}$ Queensland Tropical Health Alliance, James Cook University, Cairns, Queensland, Australia.

${ }^{2}$ Parasite Genomics Program, Department of Veterinary Science, The University of Melbourne, Melbourne, Victoria, Australia.

${ }^{3}$ Department of Microbiology, Immunology and Tropical Medicine, George Washington University, Washington, DC.

${ }^{4}$ Division of Infectious Diseases and Immunology, Queensland Institute of Medical Research, Brisbane, Queensland, Australia.

${ }^{5}$ School of Veterinary Sciences, The University of Queensland, Brisbane, Queensland, Australia.
} 
integrated approaches are needed to ensure sustainable control of schistosomiasis (Gray et al., 2010). Vaccines are an essential component for the long-term control, elimination, or eradication of infectious diseases. Here, we describe the use of various "omics" approaches to characterize the transcriptomes, genomes, proteomes, and glycomes of schistosomes and hookworms, as well as the postgenomic molecular applications of these data to identify the most suitable target antigens for vaccine development to combat the diseases they cause.

\section{The Parasites}

\section{Hookworms}

Human infections with hookworms occur predominantly in areas of rural poverty in developing countries (Hotez et al., 2004). An estimated 600-700 million people are infected with hookworms worldwide, mostly in sub-Saharan Africa, South America, and parts of Asia, notably Indonesia, Bangladesh, and India. Approximately $85 \%$ of hookworm infections are caused by Necator americanus, with the remainder caused by Ancylostoma duodenale (Hotez et al., 2004). The infective stage is the microscopic, infective larva (third-stage larva or L3), which lives in the soil until it comes in contact with human skin. Larvae actively penetrate the skin and migrate within the afferent vasculature to the lungs where they ascend the pulmonary tree to the pharynx, are swallowed, and molt to become adult male and female worms (approximately $1 \mathrm{~cm}$ in length). Adult hookworms attach to the mucosa and submucosa of the small intestine where they rupture capillaries and arterioles and feed on the extravasated blood (Brooker et al., 2004). The subsequent steps in the acquisition of the hookworm's blood meal are a major target of current vaccinomic strategies and will be discussed in this review. Almost all of the pathological effects and morbidity due to hookworm infections result from intestinal blood loss (Hotez et al., 2004). Female and male hookworms mate in the small intestine, the female releases microscopic eggs that exit the body in host feces. The eggs hatch in the environment, releasing first-stage larvae, which feed on bacteria and other organic debris in the soil before they molt twice to develop through to the ensheathed L3 stage.

\section{Schistosomes}

In contrast, schistosome flatworms (platyhelminths), also known as trematodes or flukes, cause approximately 207 million cases of human schistosomiasis worldwide, mostly in sub-Saharan Africa (Steinmann et al., 2006). However, some estimates indicate that as many as 400 million people are affected (King, 2010). In Africa, Schistosoma haematobium is the most prevalent human schistosome; it causes urinary tract schistosomiasis, comprising approximately two-thirds of the world's cases of schistosomiasis, whereas S. mansoni is the principal cause of intestinal schistosomiasis and is responsible for approximately one-third of all cases. S. mansoni also causes schistosomiasis in Latin America, with most of the cases occurring in Brazil, while S. japonicum and S. mekongi cause approximately one million cases of intestinal schistosomiasis in East Asia (Steinmann et al., 2006). Schistosomiasis is transmitted through contact with freshwater containing the infective free-swimming microscopic cercariae, which like hookworm L3, actively penetrate the skin of their human host. Cercariae that have entered human skin shed their tails to become schistosomula, which enter the vasculature and lungs before relocating to the venous system where they become sexually mature adults, pair, then mate, and egg production begins (Gryseels et al., 2006). Adults of S. haematobium migrate to the venous plexus that drains the bladder and reproductive organs, whereas, for example, S. mansoni and S. japonicum migrate to the mesenteric veins draining the intestine (Gryseels et al., 2006). Female schistosomes produce eggs, each equipped with a spine that helps to facilitate penetration through blood vessels and into the urinary tract and genitals (S. haematobium) or intestine and liver (S. mansoni and S. japonicum). Much of the pathological effects from schistosomiasis are a result of the immune response to parasite eggs trapped in host tissues during chronic infection. The resulting granulomata become fibrotic, which in turn, results in severe circulatory impairment in the affected organs (Pearce and MacDonald, 2002). In both Brazil and in sub-Saharan Africa coinfections with hookworms and schistosomes are commonly encountered (Hotez et al., 2010).

\section{Genomics and Transcriptomics}

\section{Schistosomes}

Draft genome sequences were recently published for S. mansoni and S. japonicum (Berriman et al., 2009; The Schistosoma japonicum Genome Sequencing and Functional Analysis Consortium, 2009). There is very little annotated sequence data available for $S$. haematobium, despite it being widely considered as the most important of the schistosome species in terms of prevalence and pathogenicity (Rollinson, 2009). For the purposes of this review, we will focus primarily on S. mansoni. The haploid S. mansoni genome is approximately $363 \mathrm{Mb}$ in size and contains almost 12,000 genes, distributed across seven autosomal plus sex chromosome pairs (ZW in the female and ZZ in the male) (Berriman et al., 2009). Prior to the sequencing of the genomes, comprehensive transcriptomic datasets (Verjovski-Almeida et al., 2003) and genetic maps (Criscione et al., 2009) were published, providing scaffolds with which to assemble the genome sequences. DNA microarrays have been generated by multiple groups and utilized to explore transcriptional profiles of different developmental stages of the parasite (Fitzpatrick et al., 2009; Gobert et al., 2009b, 2010; Jolly et al., 2007) (Fig. 1) and the transcriptional effects of different treatments (Gobert, 2010; Gobert et al., 2010). Fitzpatrick and colleagues (Fitzpatrick et al., 2009) conducted a thorough characterization of schistosome development using statistical and network-based exploratory analyses, and highlighted key transcriptional changes associated with life cycle progression and identified numerous candidate molecules for drug and vaccine development, including membrane spanning proteins, such as the G-protein coupled receptors and tetraspanins.

Schistosomes are acoelomate organisms (i.e., which lack a defined body cavity), and have parenchymal tissues surrounding their organs, making the manual dissection of organs or organ systems difficult. Recent advances in laser microdissection microscopy (LMM), however, has allowed researchers to start to assemble a gene atlas for schistosomes, whereby the transcriptional profiles of defined schistosome tissues or organs have been delineated using a combination of 


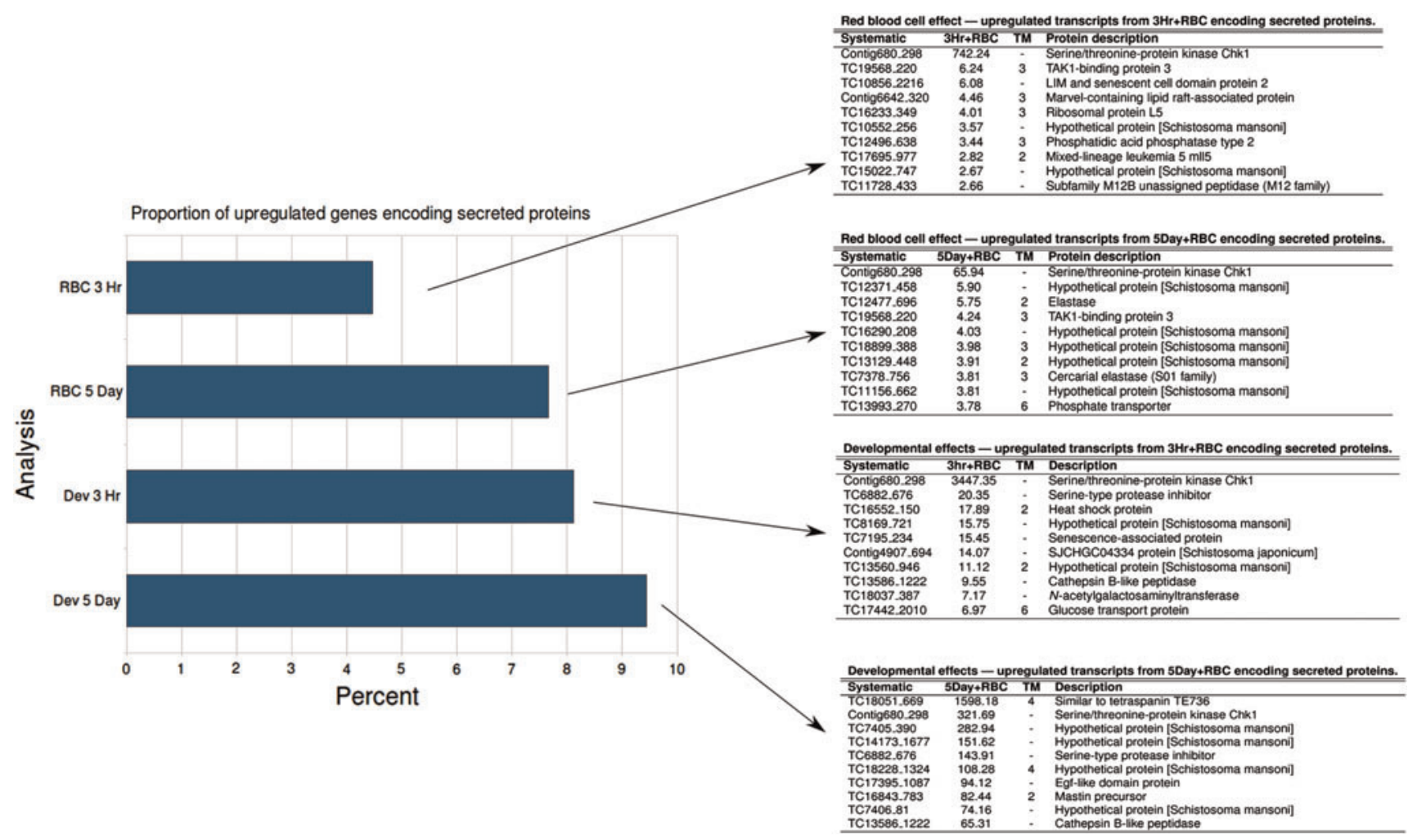

FIG. 1. Percentage of Schistosoma mansoni genes encoding for secreted/membrane proteins that underwent equal to or more than twofold increased expression in each category. Categories included 3-h-old versus 5-day-old schistosomula in the presence or absence of red blood cells (RBC). All genes that were upregulated were screened for the presence of a signal peptide or anchor using the SignalP Web server; those ORFS with a signal peptide/anchor were then further screened for transmembrane (TM) domains using TMPred. The tables show the 10 most highly upregulated genes in each category. Reproduced from Gobert et al., $2010 \mathrm{~b}$.

LMM to isolate defined tissues for RNA extraction, followed by microarray analysis to specifically identify the most abundant transcripts in tissues of interest (Gobert et al., 2009a). This approach has proven particularly relevant for the selection of antigens for vaccine and drug development. LMM has been used successfully to dissect the gut and other tissues of S. japonicum (Gobert et al., 2009a), allowing for the identification of 147 genes that were upregulated in the gastrointestinal tract of the adult female worm. Given that schistosomes reside in the vasculature and ingest blood as a source of nutrition, the gut of the parasite is considered to be a vulnerable tissue to target for vaccine development. Numerous proteolytic enzymes (proteases) were among the most highly expressed genes in gut tissues. Given their central roles in the proteolysis of haemoglobin and serum proteins (Brindley et al., 2001; Caffrey et al., 2004; Delcroix et al., 2006), these enzymes are likely to be critical for survival and, thus, worthy vaccine targets. Indeed, at least one of these proteases has been shown to be essential for the development of worms in vitro and survival in vivo using gene silencing approaches (Morales et al., 2008), more of which will be discussed later in the section on postgenomic applications. Recently, we used a microarray for $S$. mansoni to identify the most abundant transcripts in maturing schistosomula (Gobert et al., 2010), the developmental stage of the parasite that is widely considered to be the most vulnerable to the immune response against the parasite (McManus and Loukas, 2008). The most highly up- regulated genes included a tetraspanin ( $S m-t s p-3$ - one of the genes also highlighted by Fitzpatrick et al., 2009), which is known to be expressed in the outer apical membrane of the parasite (Braschi and Wilson, 2006), and was 1,600-fold upregulated during the first 5 days of schistosomula development; intestinal proteases belonging to distinct mechanistic classes were also highly upregulated, including orthologues of some proteases of $S$. japonicum that were highly expressed in gut tissue (Gobert et al., 2009a).

$S$. haemtobium is the most prevalent of the human schistosomes and is arguably the most problematic in terms of human morbidity, leading in many cases to squamous cell carcinoma of the bladder (Steinmann et al., 2006). Alarmingly, a recent report revealed that $S$. haematobium and a cattle schistosome, $S$. bovis, have hybridized in the Senegal River Basin in Africa, and hybrid parasites have been detected in human urine and feces, prompting fears of an emerging pathogen that might display hybrid vigor, including enhanced pathogenesis (Huyse et al., 2009). Despite its importance, there is a dearth of genomic and transcriptomic information for S. haematobium, hampering efforts to develop vaccines for this organism. Rollinson (2009) eloquently emphasized the need to promote research on S. haematobium in order to control it effectively, but the research community has been slow to adopt this species as the priority for research due to its reduced tractability in laboratory animal model studies. 


\section{Hookworms}

Currently, there is limited genomic sequence available for any hookworm species, although sequence surveys have been reported for the zoonotic canine hookworm, Ancylostoma caninum (Abubucker et al., 2008). However, substantial EST data exists for at least three different species-the major human hookworm, N. americanus, and the zoonotic canine hookworms, A. caninum and A. ceylanicum. Recently, deep sequencing approaches have resulted in the assembly of almost 20,000 contigs from $N$. americanus adult worm ESTs (Cantacessi et al., 2010) and up to 93\% coverage of the $A$. caninum larval and adult stage transcriptomes (Wang et al., 2010). These results have allowed the comparative analysis of the $N$. americanus and $A$. caninum transcriptomes, revealing both qualitative and quantitative differences in transcription (Cantacessi et al., 2010). For example, transcripts encoding for protease inhibitors were inferred to be highly represented in N. americanus, whereas SCP/Tpx-1/Ag5/PR-1/Sc7 (SCP/ TAPS), also referred to as Pathogenesis Related Proteins (PRPs) were predominant in A. caninum. Interestingly, the PRP family accounted for 17 of the 30 most highly upregulated genes when A. caninum L3 made the transition from freeliving to parasitic (Datu et al., 2008). Indeed, one of these PRPs from $N$. americanus, called $\mathrm{Na}$-ASP-2, is the only hookworm protein to date to have entered into a phase I clinical trial (Bethony et al., 2008).

Hookworms, like schistosomes, are relatively large, multicellular pathogens. However, unlike schistosomes, hookworms have a pseudocoelome, with easily discerned organs that float within a pressurized body cavity, which allows their removal using various traditional and microscopybased dissection approaches. LMM was used to dissect the gut and reproductive organs of $N$. americanus and A. caninum, followed by RNA extraction and construction of tissue-specific cDNA libraries for subsequent sequencing (Ranjit et al., 2006). This approach yielded a snapshot of the intestome of these hookworms and identified a suite of molecules with proven or potential roles in digestion of the blood meal, including aspartic (Loukas et al., 2005; Williamson et al., 2002) and cysteine proteases (Loukas et al., 2004), putative hemolysins (Don et al., 2004), and peptide transporters (Hotez et al., 2010). Proteins involved in the digestion of the blood meal are a major target for vaccine development within the Human Hookworm Vaccine Initiative (Hotez et al., 2010; Loukas et al., 2006; Pearson et al., 2010), and we are currently focusing on candidate molecules that have been identified using a range of "omics" approaches.

\section{Proteomics}

\section{Schistosomes}

Various tissues and fluids from schistosomes have been characterized using a range of proteomic approaches. Of particular relevance to vaccine development is the elucidation of the tegument surface proteome of the adult blood fluke. The schistosome tegument is a syncitium and acts as the direct interface between the host and the parasite, and as such, contains extracellular proteins-Skelly and Wilson (2006) have provided a highly comprehensive review on the schistosome tegument. Many vaccine antigens that are located in the tegument have been tested in murine models of schistosomiasis, but only a few are likely to be available to antibodies on the surface of a live, intact parasite. DeMarco and Verjovski-Almeida (2009) recently reviewed the use of proteomics to identify schistosome proteins for vaccine antigen and drug discovery. We have thus restricted our discussion here to studies that focus on the outer membrane proteins of the schistosome tegument, primarily because we believe that exposed membrane proteins are likely to be the most efficacious as vaccine antigens (Loukas et al., 2007; McManus and Loukas, 2008).

By labeling the surface of live adult $S$. mansoni with biotin, only primary amine groups that were exposed on the outer surface of the tegument incorporated the label, allowing for their purification via streptavidin affinity chromatography and characterization by liquid chromatography tandem mass spectrometry (LC-MS/MS) (Braschi and Wilson, 2006). Surprisingly, few proteins were detected on the surface of the live parasite, reflecting an epithelium that is relatively depauperate in surface exposed proteins. This finding, however, limits the number of target vaccine antigens to a manageable number. Indeed, where tested, these outer membrane proteins have conferred good levels of protection in mice and are recognized preferentially by antibodies from resistant people in Brazil (Cardoso et al., 2006, 2008; Tran et al., 2006). A similar study was conducted using biotinylated S. japonicum, but instead of excising bands from a onedimensional gel for protein extraction, Mulvenna and colleagues (2010) used an off-gel electrophoresis technique to minimize protein loss during sample preparation and subsequently identified orthologues of the $S$. mansoni-labeled proteins as well as additional membrane spanning proteins of interest (Fig. 2).

Parasitic helminths secrete a barrage of molecules into host tissues as they migrate, feed, and produce eggs within their hosts. One component linked to this process at the hostparasite interface is the excretory/secretory (ES) proteinsmolecules that are released from live helminths maintained in tissue culture. The presumption that proteins released in vitro mimic or at least resemble the suite of proteins released in vivo is contentious, and this is particularly evident in the ES proteome of schistosome cercariae, where of 48 spots detected on a 2D-gel, 29 were thought to originate in the secretory vesicles and 18 from the cytosol of the secretion glands, with the latter thought to be due to holocrine secretion (Curwen et al., 2006). This controversy is further highlighted by the fact that only approximately half of the proteins in the ES products from adults of S. japonicum were putatively secreted (based on their gene ontologies) and some of the most abundant proteins detected are of known intracellular origin (Liu et al., 2009).

After schistosome cercariae penetrate the skin and transform into schistosomula, they enter the vasculature and migrate (depending on species) to the portal or bladder vessels via the lungs. The first 3 to 5 days of this migratory process between the skin and the lungs is thought to represent the most susceptible stage to antibody-mediated killing (El Ridi and Tallima, 2009; McManus and Loukas, 2008; Mountford et al., 1995). Neither the tegument nor ES proteins from in vitro cultured or in vivo obtained schistosomula have been characterized to date, primarily due to the difficulty in obtaining sufficient quantities of material for such protein studies, but 


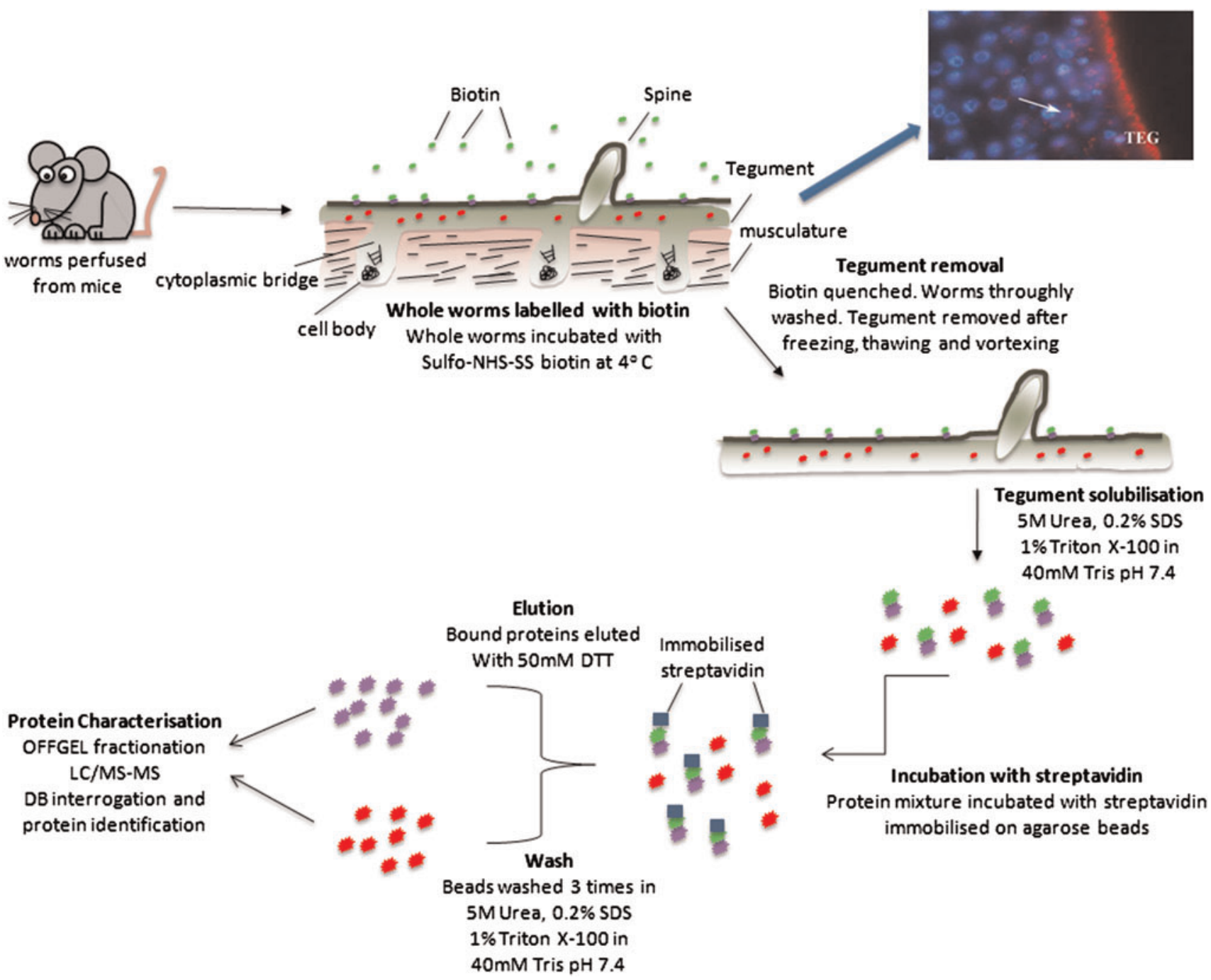

FIG. 2. Pictorial representation of the biotinylation of the surface of live Schistosoma japonicum adult worms after perfusion from mice, and subsequent processing of labeled surface proteins for proteomic analysis. The micrograph in the top right corner shows detection of the biotinylated surface of the parasite using confocal micsoscopy. The slide was stained with Cy3anti-biotin (red) and DAPI to locate nuclei (blue). Biotin was detected in the surface layer of the tegument (TEG), where it appears as a bright sinuous band of label. Positive immunoreactivity was also detected in regions subjacent to the surface layer (arrowheads), reflecting the presence of biotin in interconnecting bridges, and in discrete regions in sub-muscle cells (long arrows), likely to be tegumentary cytons. Figure modified and reproduced with permission from Mulvenna et al., 2010.

such studies are highly anticipated and would be decidedly instructive for future selection of immunogens.

\section{Hookworm ES proteome}

Schistosomes and hookworms belong to very different phyla and have distinct morphologies and physiological processes, despite sharing some similarities in biology, such as host entry (percutaneous) and feeding (on blood). Unlike ES products from schistosomes, the ES products of adult hookworms consist mostly of bona fide secreted proteins complete with signal peptide sequences. Using a strategy combining the traditional technology of one-dimensional SDS-PAGE and the newer fractionation capabilities of OFFGEL electrophoresis, 105 ES proteins were identified from the adult blood-feeding stage of A. caninum (Mulvenna et al., 2009). Highly represented among the identified proteins were carbohydrate-binding lectins and a number of proteases belonging to the three major classes: aspartic, cysteine, and metalloproteases. In hematophagous helminths, many proteases are secreted from the intestine (nematodes) or gastrodermis (trematodes), where they act to degrade host hemoglobin and serum proteins as part of the feeding process. These proteases are therefore exposed to components of the immune system of the host when the parasite ingests blood and thus represent potential targets for the development of vaccines; see review by Pearson et al. (2010).

Of the A. caninum ES proteome, $28 \%$ of the proteins identified thus far represent PRP family members (Cantacessi et al., 2009; Mulvenna et al., 2009), supporting the massive expansion of this gene family in the hookworm genome and their overrepresentation among upregulated genes in larvae during the transition to parasitism (Datu et al., 2008). Other protein families identified to date include hyaluronidases, 
lysozyme-like proteins, and transthyretin-like proteins, all of which are vaccine candidates but have yet to be assessed for efficacy in animal models.

\section{Hookworm intestome}

Targeting the hookworm gut for vaccine development has shown to be a worthwhile pursuit (Hotez et al., 2010; Loukas et al., 2006). Other blood-feeding parasites, including the barber's pole worm of livestock (Haemonchus contortus) and the cattle tick (Rhipicephalus microplus), are effectively killed by vaccine-induced antibodies against native proteins and protein complexes derived from the guts of these parasites (Smith et al., 1994). Indeed, the gut of adult $H$. contortus possesses a tightly bound multiglycoprotein complex that is rich in digestive proteases and protease inhibitors (Knox and Smith, 2001), and effective silencing of some of these genes affects parasite development, survival, and fecundity in vivo (Samarasinghe et al., 2011). It is not known whether the orthologous hookworm proteases form a similar complex to H-gal-GP, but, with the development of techniques such as LMM (Jones et al., 2004; Xu, 2010) and tissue profiling via mass spectrometry, the characterization of proteins in microscopic tissues, such as a hookworm gut, is now a possibility. Indeed, one of the lead hookworm vaccine antigens is the intestinal haemoglobinase, Na-APR-1 (a cathepsin D-like aspartic protease), where the protective effect appears to establish neutralizing antibodies that inhibits substrate proteolysis (Loukas et al., 2005; Pearson et al., 2009).

\section{Glycomics}

The role of helminth glycans as protective antigens is a complex but extremely important area. Helminths are eukaryotes and therefore decorate proteins that enter the secretory pathway with an array of glycan moieties. For a review on this topic, see Nyame et al. (2004). The difficulty in purifying or synthesizing large quantities of helminth glycans has thus far precluded their widespread use in helminth vaccine trials. However, glycans are the basis for some highly effective antibacterial vaccines (Huang and $\mathrm{Wu}, 2010$ ), and some of the most highly protective proteins from parasitic helminths are indeed glycosylated. For example, the H-galGP and H11 antigen complexes from $H$. contortus are heavily glycosylated, a feature that permitted their initial purification and identification of native protein via lectin affinity chromatographies (Knox and Smith, 2001). Where they have been characterized at the molecular level, helminth glycans offer great promise as vaccine candidates (Cummings and Nyame, 1999; Harrison et al., 2008; Maass et al., 2009).

\section{Postgenomics}

\section{Schistosomes}

One of the most revolutionary advances in the postgenomic era for the study of parasitic helminths is gene silencing by RNA interference (RNAi). Parasitic helminths, by virtue of their often complex life cycles and large genomes, have rendered themselves refractory to many genetic manipulation tools that allow the exploration of gene function (Mann et al., 2008 , 2010). RNAi is now widely used to assess gene function in schistosomes, and appears to be particularly effective for genes expressed in tissues readily accessible to dsRNA, such as the tegument and gastrodermis (Krautz-Peterson et al., 2010; Stefanic et al., 2010). RNAi has been used to confirm gene function for a number of potential schistosome vaccine antigens and drug targets, and helps explain how some vaccines, which are based on these proteins, might exert their efficacy. RNAi was used to show that the tetraspanin, Sm-TSP-2, plays important structural roles impacting tegument development, maturation or stability, and is essential for survival in vivo (Tran et al., 2010). Silencing of the genes encoding gastrodermal proteases of S. mansoni, including cathepsin D (Morales et al., 2008), papain-like cysteine proteases, and asparaginyl endopeptidase (Delcroix et al., 2006) has confirmed the roles of these proteases and the order in which they cleave their substrates during the multienzyme digestive cascade of haemoglobin. The ability to silence a schistosome gene as well as assess the effect in vitro and in vivo prompted us to suggest that the lethality of silencing a schistosome gene be one of the critical steps in ranking antigens for progress toward clinical trials for a human schistosomiasis vaccine (Hotez et al., 2010).

Its obvious utility notwithstanding, RNAi leads to only transient gene silencing and, in addition, may be inaccessible to some developmental stages and/or tissues of schistosomes. RNAi mediated by short hairpin-RNA (shRNA) expressing transgenes can induce specific and long-term knock down in eukaryotic cells (Paddison et al., 2004). In vivo, for example, vector-based, RNAi approaches that lead to integration of transgenes encoding cassettes that express short interfering RNAs can circumvent deficiencies with exogenous RNAi by providing continuous and/or conditional gene silencing (Sliva and Schnierle, 2010). In addition to vector-based RNAi, transgenesis offers the potential to endow other desirable phenotypes including the expression or coexpression of helminth or host antigens. Both vector-based RNAi and expression of foreign transgenes have begun to be deployed in studies with schistosomes, with potential outcomes including validation of putative vaccine candidates and even the overexpression of candidate antigens. Pseudotyped murine leukemia virus and the piggyBac transposon have both been shown to transduce developmental stages of schistosomes, leading to chromosomal integration of retroviral transgenes and transgene reporter activity (Kines et al., 2008; Morales et al., 2007; Yang et al., 2010). Moreover, a vector-based RNAi approach has been reported in which the MLV transgene encoded a long hairpin RNA specific for a schistosome protease involved in hemoglobinolysis (Tchoubrieva et al. 2010).

\section{Hookworms}

There are limited reports of the application of RNAi to hookworms. The technique has been used to silence (by soaking) the expression of some genes in the L3 stage of the related blood-feeding nematode, $H$. contortus (Geldhof et al., 2006; Kotze and Bagnall, 2006; Samarasinghe et al., 2011; Zawadzki et al., 2006). Successful silencing of genes in parasitic nematodes is plagued by a lack of reproducibility between and even within laboratories (Viney and Thompson, 2008). However, recently, Samarasinghe et al. (2011) showed that the proximity of the tissue expressing the mRNA of interest to the dsRNA being applied played a role in the success of RNAi in H. contortus. They showed that genes for intestinal proteases (and lead vaccine antigens) could be silenced in 
feeding third-stage larvae by soaking parasites in dsRNA. Presumably the oral uptake of this RNA afforded direct exposure to the intestinal cells expressing the mRNAs. On the other hand, genes that were expressed in tissues that were not easily accessible to ingested dsRNA were not susceptible to RNAi by soaking. The dsRNA-treated larvae were then used to infect sheep, and silencing of intestinal proteases, such as the aminopeptidase H11, resulted in reduced survival of adult worms and reduced fecundity of female worms that did survive. The ability to silence the expression of intestinal protease genes in $H$. contortus is a major advance in the area of vaccine antigen discovery for blood-feeding nematodes of livestock and humans-it allows, for the first time, an assessment of the function(s) of these proteases in vivo, and, therefore, their roles in parasite survival can potentially be addressed. For example, questions regarding functional redundancy in pathways that involve multigene families, such as the intestinal cysteine proteases of Haemonchus (Jasmer et al., 2001) and hookworms (Ranjit et al., 2008), can be addressed.

Like RNAi, transgenesis has yet to be reported in hookworms. However, progress has been reported with gene manipulation of the related parasitic nematode, Strongyloides stercoralis, in which transgene approaches developed for use in Caenorhabditis elegans have been employed to demonstrate that morphogenesis of infective L3 from earlier larval forms requires the DAF-16 orthologue FKTF-1, a forkhead transcription factor that regulates dauer larval development in $C$. elegans (Castelletto et al., 2009).

\section{Immunomics}

The availability of the $S$. mansoni and S. japonicum genomes coupled with the proteomic characterization of the tegument and ES products has provided researchers with the tools required to apply postgenomic approaches to vaccine antigen discovery. The availability of high throughput protein expression techniques, such as in vitro translation using prokaryotic or eukaryotic ribosomes, and sera from resistant humans and animals (e.g., hyperinfected rats or animals vaccinated with irradiated cercariae), means that schistosomiasis researchers are now armed and ready to utilize immunomic approaches. Recently, we designed and manufactured the first Schistosoma immunomics protein microarray (Driguez et al., 2010) and are currently using it as a vaccine discovery tool (Fig. 3). The proteins selected for inclusion on the chip includes those from previously published proteomic data and in silico screening of available sequences to identify potential immunogens based on protein location, with a particular emphasis on proteins expressed on/in the tegument. Following cloning, selected sequences were expressed in a cell-free expression system and contact-printed on to nitrocellulose coated microscope slides to form microarrays. The arrays have been probed with IgG (different subclasses) and IgE from resistant and chronically infected humans and animals (S. Gaze et al., unpublished). The approach will allow us to identify antigens that are the major target of protective IgG responses while avoiding antigens that might induce potentially harmful IgE responses when administered as vaccines (Hotez et al., 2010). This innovative technology of reverse vaccinology has the potential to transform vaccine research for schistosomiasis and other parasitic diseases of humans and animals.

The only protein microarray containing proteins derived from parasitic helminths that we are aware of is the schistosome array described above. Immunomics, however, holds great potential for accelerating antigen discovery for vaccine programs targeting other parasitic helminths, such as hookworms. Animals can be protected against hookworm disease by vaccination with irradiated L3 (Loukas et al., 2006; Miller, 1978), but only one study has attempted to identify the major targets of antibodies from this sera using a panel of only three existing recombinant antigens derived from L3 (Fujiwara et al., 2006). The transcriptomes of human and canine hookworms have been well characterized (Cantacessi et al., 2010; Wang et al., 2010), paving the way for the development of hookworm protein microarrays for antigen discovery. As for schistosomiasis vaccine antigen discovery, antigens that

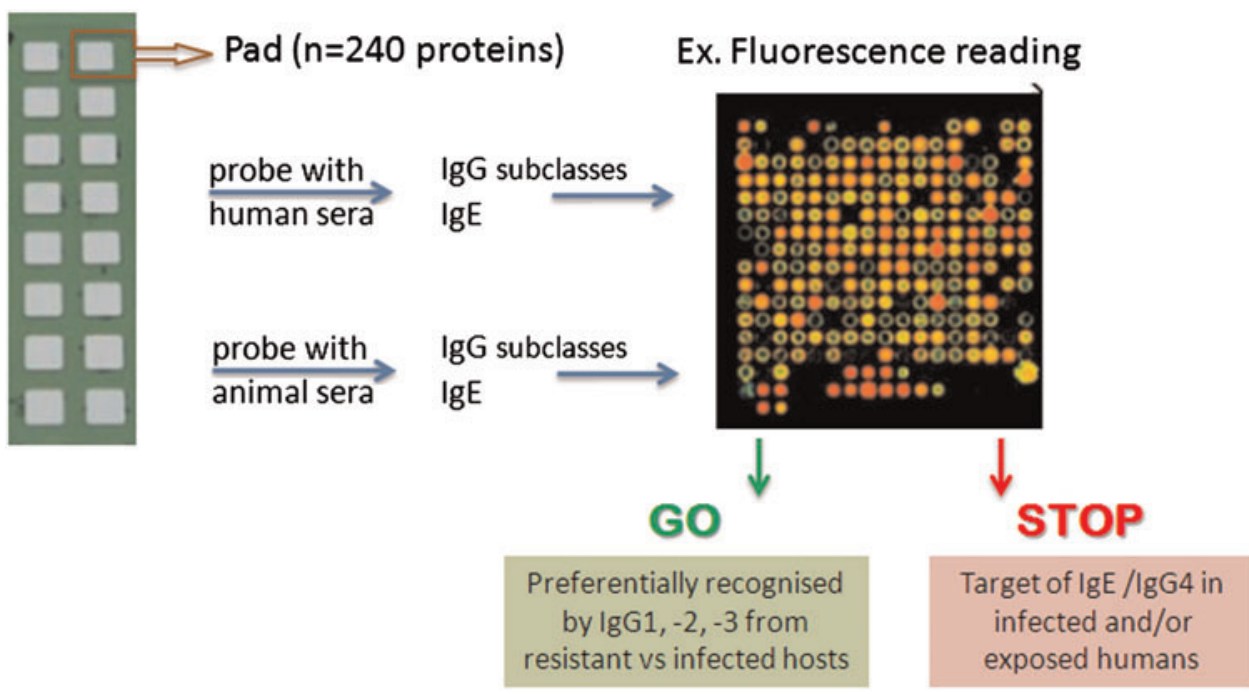

FIG. 3. Schematic depiction of the use of immunomics to develop and screen a Schistosoma protein microarray for screening with sera from resistant and chronically infected humans and animals. 
progress toward clinical trials need to be the target of a protective IgG response, while avoiding potentially deleterious IgE responses, thus warranting a pipeline of hookworm vaccine antigen discovery and production. Immunomics provides an ideal tool by which to discover and conduct early screening for up- and down-selection of proteins for progress toward clinical trials.

There are, of course, inherent problems with many highthroughput approaches, and immunomics is no exception. One of the major challenges with developing vaccines against eukaryotic pathogens, such as helminths, is the faithful replication of vaccine antigens using recombinant technologies. Given that many helminth vaccine antigens are extracellular, they are processed through the secretory pathway and often undergo complex posttranslational processing and are extensively disulfide bonded. We routinely express helminth secreted proteins in yeast or insect cells to obtain properly folded recombinant protein. Cell-free protein expression systems, whether they be components of prokaryotic or eukaryotic cells, do not possess the cellular machinery to process secreted proteins and will not always faithfully reproduce the correct fold. Nonetheless, the high-throughput nature of this expression system lends itself well to immunomic studies and has proven to be an excellent tool for identifying antigens from a range of single celled pathogens at least (Crompton et al., 2010; Davies et al., 2008; Eyles et al., 2007).

\section{Conclusions}

Major advances in biotechnology occur at a staggering pace. We can now sequence the genome and proteome of a complex pathogen in a matter of weeks or even days. For some pathogens, we can knock genes in and out of the genome and silence mRNA expression, and we can now synthesize a pathogen proteome and spot it on to a chip in an array. High throughput technology for antigen discovery no longer seems to be a barrier to developing vaccines for most pathogens, yet the number of new vaccines that have been developed during this genomic/postgenomic era is still relatively low. This might just reflect the long time required to take findings from the bench to the bedside and the funding issues that plague this "valley of death" transition. However, the (apparent) lack of success also suggests that a shotgun approach to characterizing the molecular composition of an organism is only one part of the story in vaccine development. Colossal obstacles still face vaccinologists, particularly those working on parasitic helminths. They include the difficulties in expressing large numbers of cDNAs in eukaryotic expression vectors and identifying laboratory animal models suitable for high throughput testing. For instance, hookworm antigens cannot routinely be tested in a mouse challenge model. Thus, the successes identified for reverse vaccinology approaches for bacterial vaccines against Streptococcus and Meningococcus (Rappuoli and Covacci, 2003) are not expected to promote eukaryotic parasite vaccine development anytime soon. Moreover, it is generally accepted that antihelminth vaccines will need to be multivalent (given that distinct developmental stages of helminths have very different transcriptomes and proteomes) and will require at least two shots to confer sufficient protection (Loukas et al., 2006, McManus and Loukas, 2008). Once we have used a suite of omics technologies to identify the best vaccine antigens, how do we formulate, optimize and deliver them? These are the big questions that now face us in the development of vaccines. The data and information provided through the use of "omics"technologies can now be harnessed using hypothesisdriven bench research approaches in our quest to develop as effective antischistosome and antihookworm vaccines as are currently available for the taeniid cestodes (Gauci et al., 2005).

\section{Acknowledgments}

Our work is predominantly supported by the National Health and Medical Research Council of Australia, Bill and Melinda Gates Foundation, Australian Research Council and Sabin Vaccine Institute.

\section{Author Disclosure Statement}

The authors declare that no conflicting financial interests exist.

\section{References}

Abubucker, S., Martin, J., Yin, Y., Fulton, L., Yang, S.P., Hallsworth-Pepin, K., et al. (2008). The canine hookworm genome: analysis and classification of Ancylostoma caninum survey sequences. Mol Biochem Parasitol 157, 187-192.

Albonico, M., Bickle, Q., Ramsan, M., Montresor, A., Savioli, L., and Taylor, M. (2003). Efficacy of mebendazole and levamisole alone or in combination against intestinal nematode infections after repeated targeted mebendazole treatment in Zanzibar. Bull World Health Org 81, 343-352.

Berriman, M., Haas, B.J., LoVerde, P.T., Wilson, R.A., Dillon, G.P., Cerqueira, G.C., et al. (2009). The genome of the blood fluke Schistosoma mansoni. Nature 460, 352-360.

Bethony, J.M., Bethony, J.M., Simon, G., Diemert, D.J., Parenti, D., Desrosiers, A., et al. (2008). Randomized, placebocontrolled, double-blind trial of the Na-ASP-2 hookworm vaccine in unexposed adults. Vaccine 26, 2408-2417.

Braschi, S., and Wilson, R.A. (2006). Proteins exposed at the adult schistosome surface revealed by biotinylation. Mol Cell Proteomics 5, 347-356.

Brindley, P.J., Kalinna, B.H., Wong, J.Y., Bogitsh, B.J., King, L.T., Smyth, D.J., et al. (2001). Proteolysis of human hemoglobin by schistosome cathepsin D. Mol Biochem Parasitol 112, 103-112.

Brooker, S., Bethony, J., and Hotez, P.J. (2004). Human hookworm infection in the 21st century. Adv Parasitol 58, 197-288.

Burke, W., Burton, H., Hall, A.E., Karmali, M., Khoury, M.J., Knoppers, B., et al. (2010). Extending the reach of public health genomics: what should be the agenda for public health in an era of genome-based and "personalized" medicine? Genet Med 12, 785-791.

Caffrey, C.R., McKerrow, J.H., Salter, J.P., and Sajid, M. (2004). Blood ' $\mathrm{n}$ ' guts: an update on schistosome digestive peptidases. Trends Parasitol 20, 241-248.

Cantacessi, C., Campbell, B.E., Visser, A., Geldhof, P., Nolan, M.J., Nisbet, A., et al. (2009). A portrait of the "SCP/TAPS" proteins of eukaryotes-developing a framework for fundamental research and biotechnological outcomes. Biotechnol Adv 27, 376-388.

Cantacessi, C., Mitreva, M., Jex, A.R., Young, N.D., Campbell, B.E., Hall, R.S., et al. (2010). Massively parallel sequencing and analysis of the Necator americanus transcriptome. PLoS Neglect Trop Dis 4, e684.

Cardoso, F.C., Pacifico, R.N., Mortara, R.A., and Oliveira, S.C. (2006). Human antibody responses of patients living in en- 
demic areas for schistosomiasis to the tegumental protein Sm29 identified through genomic studies. Clin Exp Immunol 144, 382-391.

Cardoso, F.C., Macedo, G.C., Gava, E., Kitten, G.T., Mati, V.L., de Melo, A.L., et al. (2008). Schistosoma mansoni tegument protein Sm29 is able to induce a Th1-type of immune response and protection against parasite infection. PLoS Negl Trop Dis 2, e308.

Castelletto, M.L., Massey, H.C., Jr., and Lok, J.B. (2009). Morphogenesis of Strongyloides stercoralis infective larvae requires the DAF-16 ortholog FKTF-1. PLoS Pathog 5, e1000370.

Clements, A.C., Bosqué-Oliva, E., Sacko, M., Landouré, A., Dembélé, R., Traoré, M., et al. (2009). A comparative study of the spatial distribution of schistosomiasis in Mali in 1984-1989 and 2004-2006. PLoS Negl Trop Dis 3, e431.

Criscione, C.D., Valentim, C.L., Hirai, H., LoVerde, P.T., and Anderson, T.J. (2009). Genomic linkage map of the human blood fluke Schistosoma mansoni. Genome Biol 10, R71.

Crompton, P.D., Kayala, M.A., Traore, B., Kayentao, K., Ongoiba, A., Weiss, G.E., et al. (2010). A prospective analysis of the $\mathrm{Ab}$ response to Plasmodium falciparum before and after a malaria season by protein microarray. Proc Natl Acad Sci USA 107, 6958-6963.

Cummings, R.D., and Nyame, A.K. (1999). Schistosome glysoconjugates. Biochim Biophys Acta 1455, 363-374.

Curwen, R.S., Ashton, P.D., Sundaralingam, S., and Wilson, R.A. (2006). Identification of novel proteases and immunomodulators in the secretions of schistosome cercariae that facilitate host entry. Mol Cell Proteomics 5, 835-844.

Datu, B., Gasser, R.B., Nagaraj, S.H., Ong, E.K., O'Donoghue, P., McInnes, R., et al. (2008). Transcriptional changes in the hookworm, Ancylostoma caninum, during the transition from a free-living to a parasitic larva. PLoS Neglect Trop Dis 2, e130.

Davies, D.H., Wyatt, L.S., Newman, F.K., Earl, P.L., Chun, S., Hernandez, J.E., et al. (2008). Antibody profiling by proteome microarray reveals the immunogenicity of the attenuated smallpox vaccine modified vaccinia virus ankara is comparable to that of Dryvax. J Virol 82, 652-663.

Delcroix, M., Sajid, M., Caffrey, C.R., Lim, K.C., Dvorák, J., Hsieh, I., et al. (2006). A multienzyme network functions in intestinal protein digestion by a platyhelminth parasite. J Biol Chem 281, 39316-39329.

DeMarco, R., and Verjovski-Almeida, S. (2009). Schistosomesproteomics studies for potential novel vaccines and drug targets. Drug Discov Today 14, 472-478.

Don, T.A., Jones, M.K., Smyth, D.J., O’Donoghue, P., Hotez, P.J., and Loukas, A. (2004). A pore-forming haemolysin from the hookworm, Ancylostoma caninum. Int J Parasitol 34, 1029-1035.

Driguez, P., Doolan, D.L., Loukas, A., Felgner, P.L., and McManus, D.P. (2010). Schistosomiasis vaccine discovery using immunomics. Parasites Vectors 3, 4.

El Ridi, R., and Tallima, H. (2009). Schistosoma mansoni ex vivo lung-stage larvae excretory-secretory antigens as vaccine candidates against schistosomiasis. Vaccine 27, 666-673.

Eyles, J.E., Unal, B., Hartley, M.G., Newstead, S.L., Flick-Smith, H., Prior, J.L., et al. (2007). Immunodominant Francisella tularensis antigens identified using proteome microarray. Proteomics 7, 2172-2183.

Fallon, P., and Doenhoff, M. (1994). Drug-resistant schistosomiasis: resistance to praziquantel and oxamniquine induced in Schistosoma mansoni in mice is drug specific. Am J Trop Med Hyg 51, 83-88.

Fitzpatrick, J.M., Peak, E., Perally, S., Chalmers, I.W., Barrett, J., Yoshino, T.P., et al. (2009). Anti-schistosomal intervention targets identified by lifecycle transcriptomic analyses. PLoS Neglect Trop Dis 3, e543.

Fujiwara, R.T., Loukas, A., Mendez, S., Williamson, A.L., Bueno, L.L., Wang, Y., et al. (2006). Vaccination with irradiated $A n$ cylostoma caninum third stage larvae induces a Th2 protective response in dogs. Vaccine 24, 501-509.

Gauci, C., Heath, D., Chow, C., and Lightowlers, M.W. (2005). Hydatid disease: vaccinology and development of the EG95 recombinant vaccine. Expert Rev Vaccines 4, 103-112.

Geerts, S., and Gryseels, B. (2000). Drug resistance in human helminths: current situation and lessons from livestock. Clin Microbiol Rev 13, 207-222.

Geldhof, P., Murray, L., Couthier, A., Gilleard, J.S., McLauchlan, G., Knox, D.P., et al. (2006). Testing the efficacy of RNA interference in Haemonchus contortus. Int J Parasitol 36, 801-810.

Gobert, G.N. (2010). Better understanding of anti-schistosomal strategies through microarray analysis. Infect Disord Drug Targets 10, 251-257.

Gobert, G.N., McManus, D.P., Nawaratna, S., Moertel, L., Mulvenna, J., and Jones, M.K. (2009a). Tissue specific profiling of females of Schistosoma japonicum by integrated laser microdissection microscopy and microarray analysis. PLoS Negl Trop Dis 3, e469.

Gobert, G.N., Moertel, L., Brindley, P.J., and McManus, D.P. (2009b). Developmental gene expression profiles of the human pathogen Schistosoma japonicum. BMC Genomics 10, 128.

Gobert, G.N., Tran, M.H., Moertel, L., Mulvenna, J., Jones, M.K., McManus, D.P., et al. (2010). Transcriptional changes in Schistosoma mansoni during early schistosomula development and in the presence of erythrocytes. PLoS Neglect Trop Dis 4, e600.

Gray, D.J., McManus, D.P., Li, Y., Williams, G.M., Bergquist, R., and Ross, A.G. (2010). Schistosomiasis elimination: lessons from the past guide the future. Lancet Infect Dis 10, 733-736.

Gryseels, B., Polman, K., Clerinx, J., and Kestens, L. (2006). Human schistosomiasis. Lancet 368, 1106-1118.

Harrison, G.B., Pulford, H.D., Doolin, E.E., Pernthaner, A., Shoemaker, C.B., and Hein, W.R. (2008). Antibodies to surface epitopes of the carbohydrate larval antigen CarLA are associated with passive protection in strongylid nematode challenge infections. Parasite Immunol 30, 577-584.

Hotez, P.J., Brooker, S., Bethony, J.M., Bottazzi, M.E., Loukas, A., and Xiao, S. (2004). Hookworm infection. New Eng J Med 351, 799-807.

Hotez, P.J., Bethony, J.M., Oliveira, S.C., Brindley, P.J., and Loukas, A. (2008a). Multivalent anthelminthic vaccine to prevent hookworm and schistosomiasis. Expert Rev Vaccines 7, 745-752.

Hotez, P.J., Brindley, P.J., Bethony, J.M., King, C.H., Pearce, E.J., and Jacobson, J. (2008b). Helminth infections: the great neglected tropical diseases. J Clin Invest 118, 1311-1321.

Hotez, P.J., Bethony, J.M., Diemert, D.J., Pearson, M., and Loukas, A. (2010). Developing vaccines to combat hookworm infection and intestinal schistosomiasis. Nat Rev Microbiol 8, 814-826.

Huang, Y.L., and Wu, C.Y. (2010). Carbohydrate-based vaccines: challenges and opportunities. Expert Rev Vaccines 9, 12571274.

Huyse, T., Webster, B.L., Geldof, S., Stothard, J.R., Diaw, O.T., Polman, K., et al. (2009). Bidirectional introgressive hybridization between a cattle and human schistosome species. PLoS Path 5, e1000571.

Jasmer, D.P., Roth, J., and Myler, P.J. (2001). Cathepsin B-like cysteine proteases and Caenorhabditis elegans homologues 
dominate gene products expressed in adult Haemonchus contortus intestine. Mol Biochem Parasitol 116, 159-169.

Jolly, E.R., Chin, C.S., Miller, S., Bahgat, M.M., Lim, K.C., DeRisi, J., et al. (2007). Gene expression patterns during adaptation of a helminth parasite to different environmental niches. Genome Biol 8, R65.

Jones, M.K., Randall, L.M., McManus, D.P., and Engwerda, C.R. (2004). Laser microdissection microscopy in parasitology: microscopes meet thermocyclers. Trends Parasitol 20, 502-506.

Keiser, J., and Utzinger, J. (2008). Efficacy of current drugs against soil-transmitted helminth infections: systematic review and meta-analysis. JAMA 299, 1937-1948.

Kines, K.J., Morales, M.E., Mann, V.H., Gobert, G.N., and Brindley, P.J. (2008). Integration of reporter transgenes into Schistosoma mansoni chromosomes mediated by pseudotyped murine leukemia virus. FASEB J 22, 2936-2948.

King, C.H. (2010). Parasites and poverty: the case of schistosomiasis. Acta Trop 113, 95-104.

King, C.H., and Dangerfield-Cha, M. (2008). The unacknowledged impact of chronic schistosomiasis. Chronic Illn 4, 65-79.

Knox, D.P., and Smith, W.D. (2001). Vaccination against gastrointestinal nematode parasites of ruminants using gutexpressed antigens. Vet Parasitol 100, 21-32.

Kotze, A.C., and Bagnall, N.H. (2006). RNA interference in Haemonchus contortus: suppression of beta-tubulin gene expression in L3, L4 and adult worms in vitro. Mol Biochem Parasitol 145, 101-110.

Krautz-Peterson, G., Bhardwaj, R., Faghiri, Z., Tararam, C.A., and Skelly, P.J. (2010). RNA interference in schistosomes: machinery and methodology. Parasitology 137, 485-495.

Liu, F., Cui, S.J., Hu, W., Feng, Z., Wang, Z.Q., and Han, Z.G. (2009). Excretory/secretory proteome of the adult developmental stage of human blood fluke, Schistosoma japonicum. Mol Cell Proteomics 8, 1236-1251.

Loukas, A., Bethony, J.M., Williamson, A.L., Goud, G.N., Mendez, S., Zhan, B. et al. (2004). Vaccination of dogs with a recombinant cysteine protease from the intestine of canine hookworms diminishes the fecundity and growth of worms. J Infect Dis 189, 1952-1961.

Loukas, A., Bethony, J.M., Mendez, S., Fujiwara, R.T., Goud, G.N., Ranjit, N., et al. (2005). Vaccination with recombinant aspartic hemoglobinase reduces parasite load and blood loss after hookworm infection in dogs. PLoS Med 2, e295.

Loukas, A., Bethony, J., Brooker, S., and Hotez, P. (2006). Hookworm vaccines: past, present, and future. Lancet Infect Dis 6, 733-741.

Loukas, A., Tran, M., and Pearson, M.S. (2007). Schistosome membrane proteins as vaccines. Int J Parasitol 37, 257-263.

Maass, D.R., Harrison, G.B., Grant, W.N., Hein, W.R., and Shoemaker, C.B. (2009). Intraspecific epitopic variation in a carbohydrate antigen exposed on the surface of Trichostrongylus colubriformis infective L3 larvae. PLoS Pathog 5, e1000597.

Mann, V.H., Morales, M.E., Kines, K.J., and Brindley, P.J. (2008). Transgenesis of schistosomes: approaches employing mobile genetic elements. Parasitology 135, 141-153.

Mann, V.H., Morales, M.E., Rinaldi, G., and Brindley, P.J. (2010). Culture for genetic manipulation of developmental stages of Schistosoma mansoni. Parasitology 137, 451-462.

McManus, D.P., and Loukas, A. (2008). Current status of vaccines for schistosomiasis. Clin Microbiol Rev 21, 225-242.

Miller, T.A. (1978). Industrial development and field use of the canine hookworm vaccine. Adv Parasitol 16, 333-342.

Morales, M.E., Mann, V.H., Kines, K.J., Gobert, G.N., Fraser, M.J., Jr., Kalinna, B.H., et al. (2007). piggyBac transposon mediated transgenesis of the human blood fluke, Schistosoma mansoni. FASEB J 21, 3479-3489.

Morales, M.E., Rinaldi, G., Gobert, G.N., Kines, K.J., Tort, J.F., and Brindley, P.J. (2008). RNA interference of Schistosoma mansoni cathepsin D, the apical enzyme of the hemoglobin proteolysis cascade. Mol Biochem Parasitol 157, 160-168.

Mountford, A.P., Harrop, R., and Wilson, R.A. (1995). Antigens derived from lung-stage larvae of Schistosoma mansoni are efficient stimulators of proliferation and gamma interferon secretion by lymphocytes from mice vaccinated with attenuated larvae. Infect Immun 63, 1980-1986.

Mulvenna, J., Hamilton, B., Nagaraj, S.H., Smyth, D., Loukas, A., and Gorman, J.J. (2009). Proteomics analysis of the excretory/ secretorycomponent of the blood-feeding stage of the hookworm, Ancylostoma caninum. Mol Cell Proteomics 8.1, 109-121.

Mulvenna, J., Moertel, L., Jones, M.K., Nawaratna, S., Lovas, E.M., Gobert, G.N., et al. (2010). Exposed proteins of the Schistosoma japonicum tegument. Int J Parasitol 40, 543-554.

Nyame, A.K., Kawar, Z.S., and Cummings, R.D. (2004). Antigenic glycans in parasitic infections: implications for vaccines and diagnostics. Arch Biochem Biophys 426, 182-200.

Paddison, P.J., Cleary, M., Silva, J.M., Chang, K., Sheth, N., Sachidanandam, R., et al. (2004). Cloning of short hairpin RNAs for gene knockdown in mammalian cells. Nat Methods 1, 163-167.

Pearce, E.J., and MacDonald, A.S. (2002). The immunobiology of schistosomiasis. Nat Rev Immunol 2, 499-511.

Pearson, M.S., Bethony, J.M., Pickering, D.A., de Oliveira, L.M., Jariwala, A., Santiago, H., et al. (2009). An enzymatically inactivated hemoglobinase from Necator americanus induces neutralizing antibodies against multiple hookworm species and protects dogs against heterologous hookworm infection. FASEB J 23, 3007-3019.

Pearson, M.S., Ranjit, N., and Loukas, A. (2010). Blunting the knife: development of vaccines targeting digestive proteases of blood-feeding helminth parasites. Biol Chem 391, 901-911.

Ranjit, N., Jones, M.K., Stenzel, D.J., Gasser, R.B., and Loukas, A. (2006). A survey of the intestinal transcriptomes of the hookworms, Necator americanus and Ancylostoma caninum, using tissues isolated by laser microdissection microscopy. Int J Parasitol 36, 701-710.

Ranjit, N., Zhan, B., Stenzel, D.J., Mulvenna, J., Fujiwara, R., Hotez, P.J., et al. (2008). A family of cathepsin B cysteine proteases expressed in the gut of the human hookworm, Necator americanus. Mol Biochem Parasitol 160, 90-99.

Rappuoli, R., and Covacci, A. (2003). Reverse vaccinology and genomics. Science 302, 602.

Rollinson, D. (2009). A wake up call for urinary schistosomiasis: reconciling research effort with public health importance. Parasitology 136, 1593-1610.

Samarasinghe, B., Knox, D.P., and Britton, C. (2011). Factors affecting susceptibility to RNA interference in Haemonchus contortus and in vivo silencing of an $\mathrm{H} 11$ aminopeptidase gene. Int J Parasitol 41, 51-59.

Skelly, P.J., and Wilson, R.A. (2006). Making sense of the schistosome surface. Adv Parasitol 63, 185-284.

Sliva, K., and Schnierle, B.S. (2010). Selective gene silencing by viral delivery of short hairpin RNA. Virol J 7, 248.

Smith, W.D., Smith, S.K., and Murray, J.M. (1994). Protection studies with integral membrane fractions of Haemonchus contortus. Parasite Immunol 16, 231-241.

Stefanic, S., Dvorak, J., Horn, M., Braschi, S., Sojka, D., Ruelas, D.S., et al. (2010). RNA interference in Schistosoma mansoni schistosomula: selectivity, sensitivity and operation for largerscale screening. PLoS Negl Trop Dis 4, e850. 
Steinmann, P., Keiser, J., Bos, R., Tanner, M., and Utzinger, J. (2006). Schistosomiasis and water resources development: systematic review, meta-analysis, and estimates of people at risk. Lancet Infect Dis. 6, 411-425.

Tchoubrieva, E.B., Ong, P.C., Pike, R.N., Brindley, P.J., and Kalinna, B.H. (2010). Vector-based RNA interference of cathepsin B1 in Schistosoma mansoni. Cell Mol Life Sci 67, 3739-3748.

The Schistosoma japonicum Genome Sequencing and Functional Analysis Consortium. (2009). The Schistosoma japonicum genome reveals features of host-parasite interplay. Nature 460, 345-352.

Tran, M.H., Pearson, M.S., Bethony, J.M., Smyth, D.J., Jones, M.K., Duke, M., et al. (2006). Tetraspanins on the surface of Schistosoma mansoni are protective antigens against schistosomiasis. Nat Med 12, 835-840.

Tran, M.H., Freitas, T.C., Cooper, L., Gaze, S., Gatton, M.L., Jones, M.K., et al. (2010). Suppression of mRNAs encoding tegument tetraspanins from Schistosoma mansoni results in impaired tegument turnover. PloS Path 6, e1000840.

Verjovski-Almeida, S., DeMarco, R., Martins, E.A., Guimaraes, P.E., Ojopi, E.P., Paquola, A.C., et al. (2003). Transcriptome analysis of the acoelomate human parasite Schistosoma mansoni. Nat Genet 35, 148-157.

Viney, M.E., and Thompson, F.J. (2008). Two hypotheses to explain why RNA interference does not work in animal parasitic nematodes. Int J Parasitol 38, 43-47.

Wang, Z., Abubucker, S., Martin, J., Wilson, R.K., Hawdon, J., and Mitreva, M. (2010). Characterizing Ancylostoma caninum transcriptome and exploring nematode parasitic adaptation. BMC Genomics 11, 307.

Williamson, A.L., Brindley, P.J., Abbenante, G., Prociv, P., Berry, C., Girdwood, K., et al. (2002). Cleavage of hemoglobin by hookworm cathepsin D aspartic proteases and its potential contribution to host specificity. FASEB J 16, 1458-1460.

$\mathrm{Xu}$, B.J. (2010). Combining laser capture microdissection and proteomics: methodologies and clinical applications. Proteomics Clin Appl 4, 116-123.

Yang, S., Brindley, P.J., Zeng, Q., Li, Y., Zhou, J., Liu, Y., et al. (2010). Transduction of Schistosoma japonicum schistosomules with vesicular stomatitis virus glycoprotein pseudotyped murine leukemia retrovirus and expression of reporter human telomerase reverse transcriptase in the transgenic schistosomes. Mol Biochem Parasitol 174, 109-116.

Zawadzki, J.L., Presidente, P.J., Meeusen, E.N., and De Veer, M.J. (2006). RNAi in Haemonchus contortus: a potential method for target validation. Trends Parasitol 22, 495-499.

Address correspondence to: Alex Loukas, Ph.D.

Queensland Tropical Health Alliance James Cook University Building E1

McGregor Rd, Smithfield Cairns QLD 4878, Australia

E-mail: Alex.Loukas@jcu.edu.au 
